Note: this is a draft of the journal article:

Dickinson, A.S., Browne, M., Roques, A.C., Taylor, A.C. (2013) "A Fatigue Assessment Technique for Modular and Pre-Stressed Orthopaedic Implants”. Medical Engineering and Physics, 36(1) 72-80, DOI: 10.1016/j.medengphy.2013.09.009

The final, fully proofed and peer-reviewed journal article is available from the publisher online, via the following link: http://www.sciencedirect.com/science/article/pii/S135045331300218X 


\title{
A FATIGUE ASSESSMENT TECHNIQUE FOR MODULAR AND PRE-STRESSED ORTHOPAEDIC IMPLANTS
}

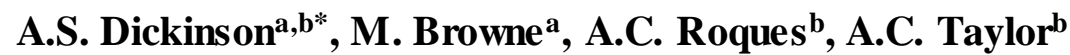 \\ a University of Southampton, United Kingdom \\ ${ }^{\mathrm{b}}$ Aurora Medical Ltd., United Kingdom
}

* Corresponding Author.

Address:

5/3019, Bioengineering Science Research Group,

University of Southampton,

Highfield,

Southampton,

SO17 1BJ

United Kingdom

Email:

alex.dickinson@soton.ac.uk

Telephone:

00442380592443

Fax:

00442380593016

Keywords :

Titanium, Fatigue, Soderberg, Arthroplasty, Preclinical Analysis 


\begin{abstract}
Orthopaedic implants experience large cyclic loads, and pre-clinical analysis is conducted to ensure they can withstand millions of loading cycles. Acetabular cup developments aim to reduce wall thickness to conserve bone, and this produces high pre-stress in modular implants. As part of an implant development process, we propose a technique for preclinical fatigue strength assessment of modular implants which accounts for this mean stress, stress concentrating features and material processing.
\end{abstract}

A modular cup's stress distributions were predicted computationally, under assembly and in-vivo loads, and its cyclic residual stress and stress amplitude were calculated. For verification against damage initiation in low-cycle-fatigue (LCF), the peak stress was compared to the material's yield strength. For verification against failure in high-cyclefatigue $(\mathrm{HCF})$ each element's reserve factor was calculated using the conservative Soderberg infinite life criterion.

Results demonstrated the importance of accounting for mean stress. The cup was predicted to experience high cyclic mean stress with low magnitude stress amplitude: a low cyclic load ratio $\left(R_{l}=0.1\right)$ produced a high cyclic stress ratio $\left(R_{s}=0.80\right)$. Furthermore the locations of highest cyclic mean stress and stress amplitude did not coincide. The minimum predicted reserve factor $N_{f}$ was $1.96(\mathrm{HCF})$ and 2.08 (LCF). If mean stress were neglected or if the stress ratio were assumed to equal the load ratio, the reserve factor would be considerably lower, potentially leading to over-engineering, reducing bone conservation.

Fatigue strength evaluation is only one step in a broader development process, which should involve a series of verifications with the full range of normal and traumatic physiological loading scenarios, with representative boundary conditions and a representative environment. This study presents and justifies a fatigue analysis methodology which could be applied in early stage development to a variety of modular and pre-stressed prosthesis concepts, and is particularly relevant as implant development aims to maximise modularity and bone conservation. 


\section{INTRODUCTION}

The fracture and fatigue strength of novel joint replacement implants should be verified against the full range of life cycle loading. This includes impact and cyclic loads several times the weight of the body, as a result of normal activities [1] and occasional traumatic events such as stumbles and falls [2]. With over one million high magnitude load cycles per year [3-5] fatigue must be a primary focus in pre-clinical analysis and testing.

Recent acetabular cup implant developments have aimed to reduce wall thickness, which permits an increase in bearing diameter without requiring additional bone removal during surgery [6-8]. A large bearing diameter promotes joint stability and reduces the risk of dislocation $[9,10]$, allowing patients to return to an active lifestyle. Bone stock preservation is beneficial later, if the implant should need to be revised [11]. However, fatigue strength verification will become particularly important, because reducing the implant's section thickness will increase its cyclic stress.

Modular acetabular cups comprise a ceramic or polymeric bearing insert for low wear articulation, and a titanium alloy (Ti-6Al-4V) outer shell featuring a rough, bioactive Hydroxyapatite (HA) coating to promote cementless fixation through osseointegration (Figure 1, left). Modular assembly of the insert and shell by a tapered interference fit enables a range of implant size options. In ceramic bearing cups, the interference fit generates a compressive residual stress field in the bearing shell rim, which is protective against ceramic fracture under tensile stresses generated under in-vivo loading [7]. Thinner pre-stressed shells (Figure 1, right) also produce a greater push-out strength at the taper interface than is achieved with a thicker, stiffer shell [8], theoretically reducing the risk of insertshell dissociation in-vivo $[12,13]$. However, this will also generate higher residual tensile stress in the titanium alloy shell.
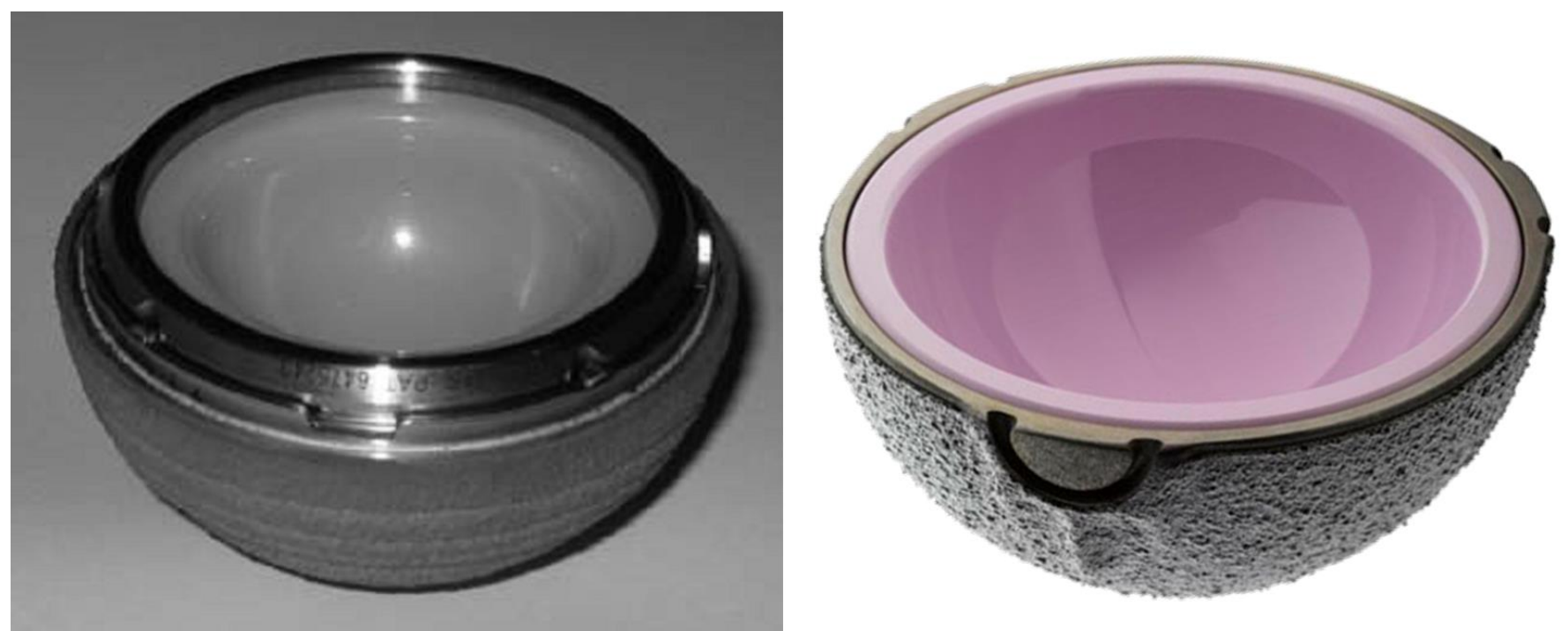

Figure 1: Example Traditional, Thick-Walled Ceramic Cup (Trident ${ }^{\circledR}$, Stryker, Mahwah, NJ, reproduced with permission and copyright $\odot$ of the British Editorial Society of Bone and Joint Surgery [13], left) and Current, ThinWalled Cup (DeltaMotion $®$, DePuy International, Warsaw, IN).

Standard ISO and ASTM endurance test methods exist for traditional implant designs, but a challenge to the bioengineer is the development of clinically representative in-vitro tests and computational analysis methods for novel designs. The same applies to computational studies. Traditional analysis of fatigue strength compares the material's endurance limit to the peak cyclic stress, and expresses the ratio as the reserve factor $\left(N_{f}\right)$. A design is judged safe under a given load regime if the reserve factor is greater than 1 , and this may be an appropriate simplification if the fatigue stress ratio $\left(R_{s}\right)$ is close to zero. The high residual stress scenario in thin walled modular implants would be expected to produce high mean stress and low stress amplitude conditions (a high stress-ratio fatigue cycle), for which this traditional fatigue analysis method may be inadequate. Furthermore, there are geometric design features and manufacturing processes that can alter the fatigue life of an implant. Any analysis should also include the influence of stress-concentrating design features such as fins for primary cup stability, and for attachment to surgical instruments for cup introduction, impaction, re-positioning and removal (Figure 1). This is of particular importance in titanium alloy components, which are notch sensitive [14-16]. The influence of manufacturing and processing upon the fatigue strength of the materials should also be considered, such as the 
incorporation of coatings which are essential for cementless fixation but have been observed to reduce the material's endurance limit [17]. If these factors are neglected, analysis may over-estimate a design's reserve factor against fatigue damage initiation. If these factors are accounted for, the traditional approach mentioned above may underestimate the true fatigue reserve factor, leading to over engineering. In the case of an acetabular cup, this would require increasing the wall thickness at the expense of bone conservation, potentially compromising the patient in future surgery.

The objective of this study was to consider increasingly challenging implant development requirements, and propose a pre-clinical fatigue strength analysis process for novel modular implant concepts. Using this process, thinner walled implants may be developed that retain a reserve factor against fatigue damage initiation under in-vivo loads, by accounting for residual and cyclic stresses, and stress concentrating features and material processing methods. 


\section{$2.1 \quad$ Case Study Implant Design Details}

The pre-clinical analysis methodology was applied to a thin walled, pre-stressed acetabular cup concept as a case study. The study employed implant geometry and (commercially confident) design details representative of a prototype variant of a modular ceramic bearing acetabular cup now in clinical use [7, 8]. The worst-case of the full range of prosthesis size options in terms of component stresses was selected for analysis, which was the thinnest walled option, featuring a nominal $5 \mathrm{~mm}$ combined wall thickness of the ceramic bearing and titanium alloy shell components.

\section{$2.2 \quad$ FE Analysis Methodology}

Finite element (FE) analysis was used to predict the stresses in the implant components under assembly and a range of clinical loading scenarios. The implant geometry (Figure 2a) was drawn in SolidWorks CAD software (SolidWorks Corp., Concord, MA, USA) imported into ANSYS 14 FE analysis software (ANSYS Inc., Canonsburg, PA, USA), map-meshed with 13,200 twenty-node hexahedral elements (64,612 nodes), and its two components were assigned linear elastic material properties (Table 1) [18]. A Coulomb slip-stick friction model was used to simulate contact between the insert and shell, with a coefficient of static friction of 0.32 (Appendix 2), determined by empirical fit to experimental data. Contact employed 8-node surface-to-surface contact and target elements, with an Augmented Lagrangian contact algorithm.

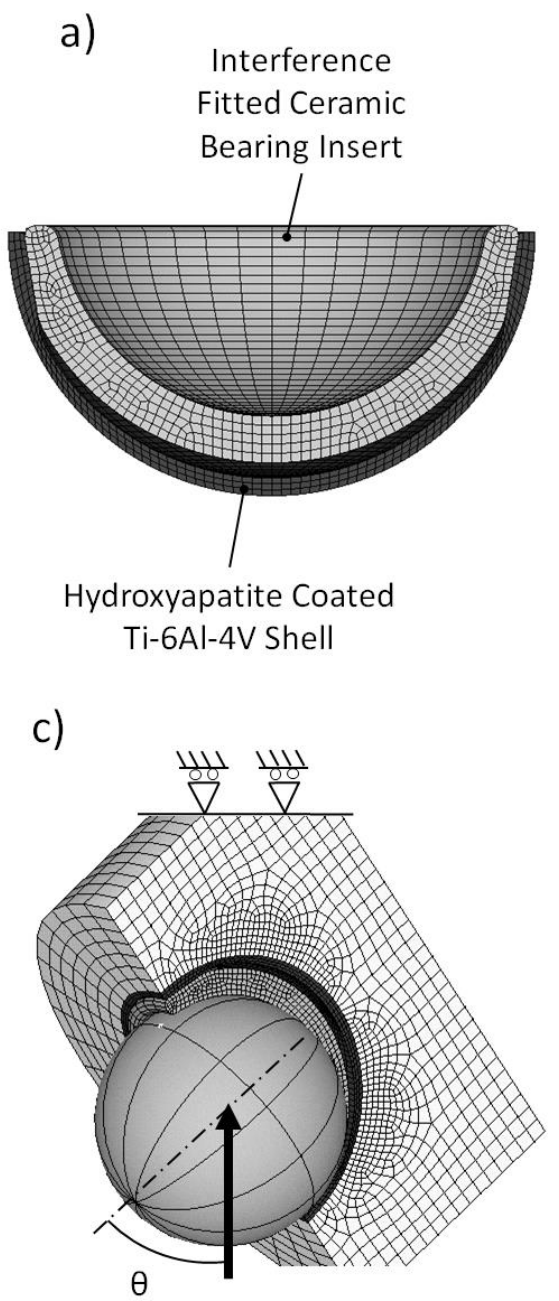

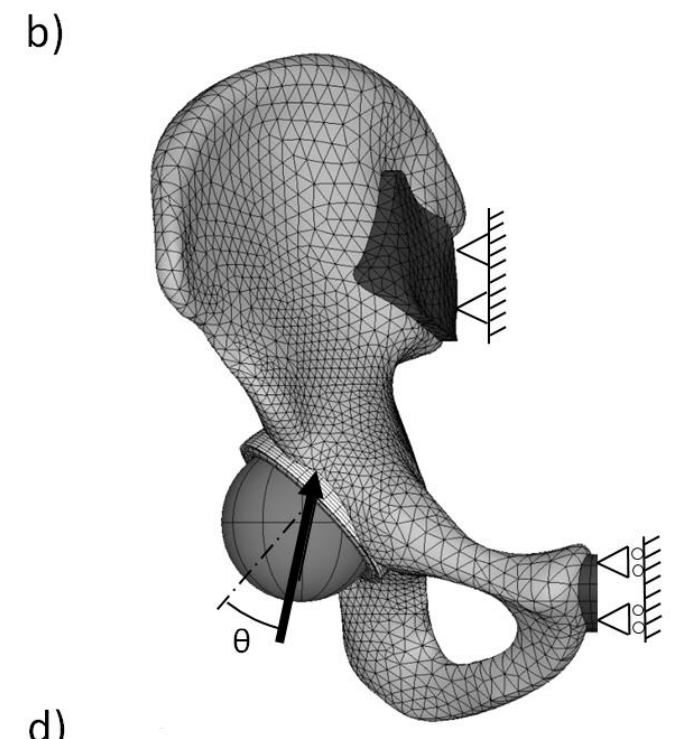

d)

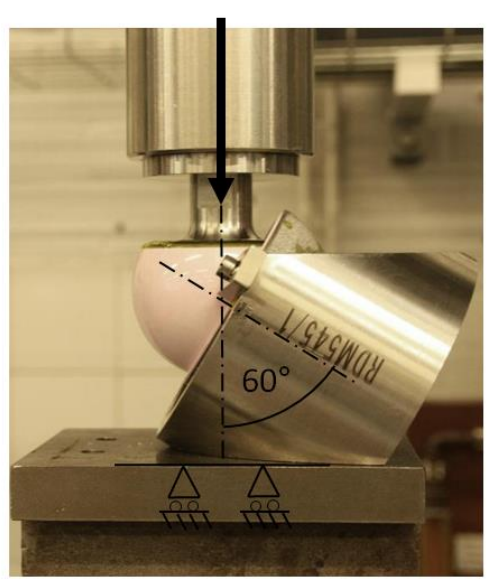

e)

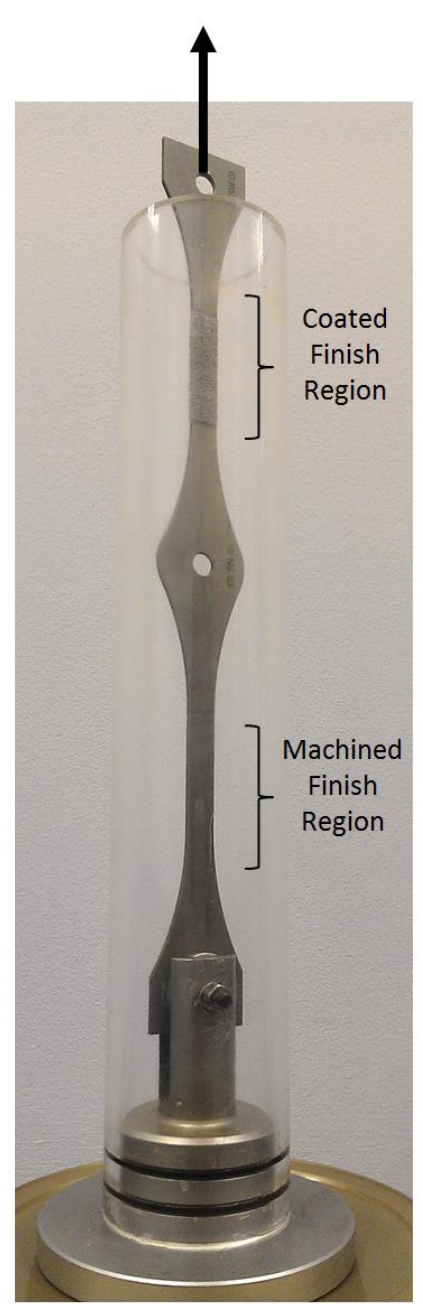

Figure 2: Modelling and Testing Arrangements. a) FE Model Schematic of Acetabular Cup Components, b) FE Model of Assembled Cup Implanted in Composite Hemi-Pelvis, c) FE Model of Assembled Cup Implanted in PMMA Support, and d) Physical Test Setup with PMMA Support. e) Representative Material Strength and Endurance Data Test Setup with Environmental Chamber 
Table 1: Assigned Material Properties

\begin{tabular}{|c|c|c|c|}
\hline Model Region & Material & $\begin{array}{l}\text { Young's } \\
\text { Modulus (GPa) }\end{array}$ & $\begin{array}{l}\text { Pois son's } \\
\text { Ratio }\end{array}$ \\
\hline Cup Bearing Insert ${ }^{a}$ & $\begin{array}{l}\text { BIOLOX Delta } \\
\text { (CeramTec AG, } \\
\text { Plochingen, Germany) }\end{array}$ & 350 & 0.22 \\
\hline Cup Outer Shell $^{\mathrm{b}}$ & Ti-6Al-4V ELI Alloy & 114 & 0.34 \\
\hline \multirow{2}{*}{ Analogue Bone } & $\begin{array}{l}\text { Cortical Bone: } \\
\text { Short Glass Fibre Reinforced Epoxy } \\
\text { (Sawbones AG, Malmo, Sweden) }\end{array}$ & 16.7 & 0.3 \\
\hline & $\begin{array}{l}\text { Cancellous Bone: } \\
\text { Polyurethane Foam } \\
\text { (Sawbones AG, Malmo, Sweden) }\end{array}$ & 0.155 & 0.3 \\
\hline Test Support Material $^{\mathrm{d}}$ & $\begin{array}{l}\text { PMMA } \\
\text { (Technovit } ® 3040, \\
\text { Heraeus GmbH, Hanau, Germany) }\end{array}$ & 2.3 & 0.35 \\
\hline
\end{tabular}

\footnotetext{
${ }^{a}$ http://www.ceramtec.com/files/mt_biolox_delta_en.pdf p11[18,33]

b ASTM F136-12a [18,32]

${ }^{\mathrm{c}}$ http://www.sawbones.com/catalog/pdf/us catalog.pdf p $75[18,19]$

$\mathrm{d}$ http://heraeus-kulzer-technik.de/media/webmedia local/media/metallo/downloads 1/BR Technovit englisch.pdf p24 [20]
}

The cup was modelled as supported in two ways:

- first, implanted in a generic hemi-pelvis geometric model (Zygote Inc., UT, USA) with material properties and cortical shell thickness representing a composite analogue hemi-pelvis model (\#3405, Sawbone AG, Malmö, Sweden [19]), fixed in all directions at the sacro-iliac joint, and in the medial-lateral direction at the pubic symphysis (Figure $2 \mathrm{~b}$ ), and

- second, in a laboratory test setup, with the cup mounted in a bed of PMMA potting medium (Technovit 3040 polymethyl methacrylate, Heraeus Kulzer GmbH, Hanau, Germany [20]), fixed at its base (Figure 2c).

In both cases, the cup was modelled as fully bonded to the support structure. 7,660 hexahedral elements $(34,160$ nodes) were used to mesh the PMMA support, and 36,644 tetrahedral solid and triangular shell elements (56,143 nodes) were used to mesh the hemi-pelvis. The support models were assigned homogeneous linear elastic material properties (Table 1). The hemi-pelvis model (total solution time $\sim 12$ hours) was used to obtain baseline results to verify the PMMA support of the laboratory test model (total solution time $\sim 2$ hours), which was then used with a considerably finer mesh in order to capture a contact stress peaks at the taper interface. These solution times were obtained using a sparse matrix solver, running in-core on a 3GHz, 8GB RAM desktop machine.

A range of surgical positioning was assessed by solving the models for a range of cup inclination angles, of $40^{\circ}$ (ideal), $55^{\circ}$ and $70^{\circ}$ (extreme), all with $20^{\circ}$ anteversion. This was achieved directly through cup positioning in the hemi-pelvis models, and indirectly, through alignment of the load vector in the PMMA models. Incorporation of a $10^{\circ}$ adducted joint contact force vector in the hemipelvis models [1] gave an angle between the force vector and the cup axis of $34.9^{\circ}, 47.7^{\circ}$ and $61.3^{\circ}$ for the three models, respectively. A range of joint contact force magnitudes was applied to represent different loading scenarios, justified below.

\subsection{Loading Scenarios}

Two loads were applied to each model, representing press fitted assembly, and a superimposed cyclic in-vivo activity load. Assembly was simulated using displacement control to press the ceramic insert by its rim into the metal shell, supported at its pole [7]. The assembly press was then removed, and coulomb friction at the taper interface maintained the press-fit and the resulting initial pre-stress in the implant.

For high cycle fatigue analysis, gait loading was simulated. A joint contact force of $5.8 \mathrm{kN}$ was applied to the implant bearing surface through a single, rigid spherical head with frictionless contact. The effects of articulation and bearing friction were considered separately as described in Section 2.4 , local to the cup fixation fins, where the resulting torque would be concentrated. The applied force represents the maximum 584\% bodyweight load recorded during jogging from instrumented implant clinical studies [21, 22], which is a likely worst-case cyclic normal activity load. Hip replacement patients are reported to exert 1-2.5 million gait cycles per year [3-5, 22] upon each lower limb joint. Although the majority of these cycles are walking loads, jogging represents a worst-case. 
For low cycle fatigue analysis, abnormal or traumatic loads were considered and an $11 \mathrm{kN}$ joint contact force was applied to the head, to represent stumbling or falling. Stumbling is the highest magnitude, commonly occurring load event experienced at the hip joint during articulation [2, 22], and sideways falling onto the hip is a typical high magnitude non-articulating load event. The loads resulting from falling are more variable; peak forces are reported to reach 8-11 times bodyweight [23, 24], depending upon fall velocity, soft tissue thickness and muscle activity [25]. One in three elderly patients experiences a fall in a given year [26], and stumbling without falling is reported to occur two or more times per month in $9 \%$ of this population. For a young patient, 10 stumbles per month is estimated [22]. Hence, during a 20 year implant life, an implant may experience in the order of 2500 stumbles and 7 falls. Therefore, these load events can be considered as potential low-cycle fatigue loads.

\section{$2.4 \quad$ Fatigue Analysis Methodology A: High Cycle Fatigue Loads}

The assembly loading was predicted to generate a residual tensile stress in the titanium shell; above this, the in-vivo joint contact force was predicted to create additional cyclic tension. The predictions of the FE model were therefore analysed against an infinite life limit for elastic-plastic materials. Several of these criteria have been established, by Gerber in 1874 [27], Goodman in 1899 [28], and the most conservative, selected for this study, by Soderberg in 1930 [29].

The fatigue reserve factor $N_{f}$ versus the Soderberg infinite life limit was calculated as described in Appendix 1 for each node in the titanium alloy shell. Four sets of parameters were used in the calculation, to represent different surface finishes and stress concentrations on the cup surfaces (Figure 3):

a) machined finish on the shell's internal surfaces without stress concentrations,

b) grit blasted and hydroxyapatite (HA) coated finish on the shell's external surface without stress concentrations,

c) grit blasted and HA-coated finish in a band around the shell's rim, with additional cyclic stress resulting from the reaction against torque in the bearing at the shell's anti-rotation fins, and

d) machined finish in a band around the shell's rim, with additional stress concentration resulting from geometric features for attachment of the cup's introducer instrument.

Machined surfaces were assumed to have a roughness of $\mathrm{R}_{\mathrm{a}} \approx 0.8 \mu \mathrm{m}$, and grit blasted surfaces a roughness of $\mathrm{R}_{\mathrm{a}} \approx$ $3.6 \mu \mathrm{m}$ [17]. In the band around the shell's rim, which contained regions of material in both surface conditions $\mathrm{c}$ and $\mathrm{d}$, the surface condition which produced the lower reserve factor value was used.

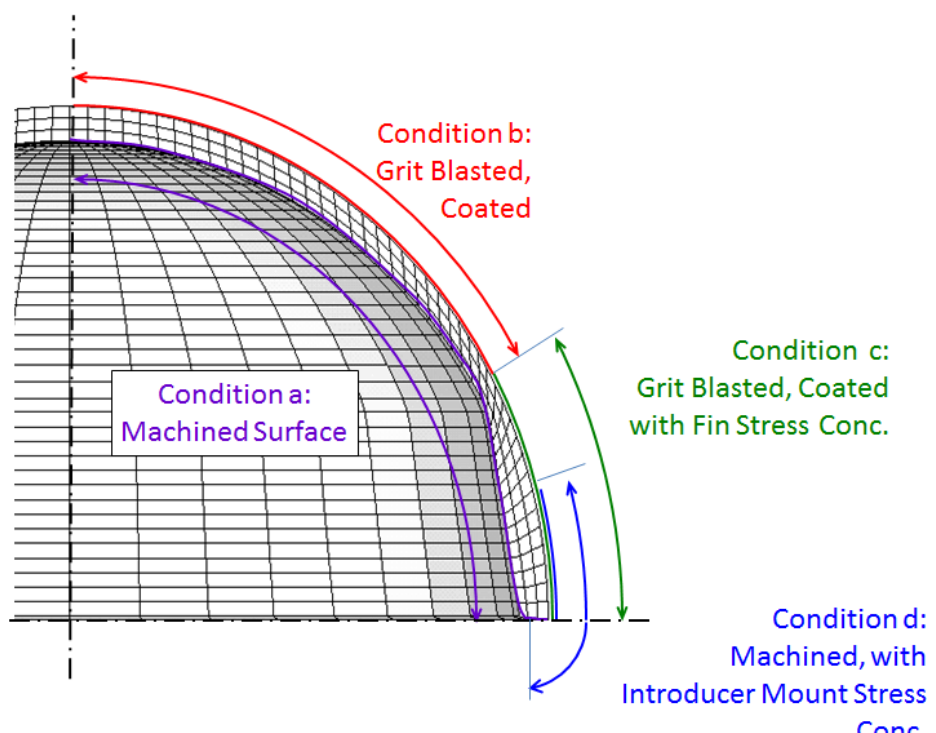

Figure 3: Schematic View of Titanium Shell showing Different Surface Condition Regions for Manufacturing Processes, and Stress Concentrations from Anti-Rotation Fins and Introducer Mounting Features. Not to Scale.

For all cup regions featuring material conditions a-d, each node's cyclic mean stress and stress amplitude were calculated from the residual $\left(\sigma_{\text {assembly }}\right)$ and peak cyclic $\left(\sigma_{\text {jogging }}\right)$ load cases, accounting for the fatigue load ratio $R_{L}$ (Appendix 1). Over the jogging cycle the cyclic load component varies between approximately $10 \%$ and $100 \%$ of the peak load [21], so a value of $R_{L}=0.1$ was used. For condition c, the additional cyclic stress resulting from the 
reaction of the bearing's frictional torque by the shell's fins (Figure 1) was added to the stress amplitude for all nodes in a band region around the rim of the shell on its external surface (Figure 3). This additional stress amplitude was determined us ing a sub-model of the shell featuring a tangentially loaded fin (not reported). The tangential load was determined using a friction factor of 0.05 , the maximum measured for the bearings in question in a simulator study by Scholes and Unsworth [30], and exerting the bearing frictional torque that this generated over a single fin.

The influence of stress concentrating geometric features in high cycle fatigue was incorporated in the fatigue stress concentration factor $K_{f}$ (Appendix 1), as a function of the stress concentration factor $K_{t}$, which accounts for the stress concentrating effect of geometric features in the structure. For conditions a-c, without geometric stress concentrations, the minimum $K_{t}=1$ was used. For condition d, in the region containing the shell's introducer attachment features, $K_{t}$ was conservatively estimated from the results of a sub-model of a section of the shell featuring an introducer attachment wire groove (as in Figure 1, not reported), from the ratio of the local peak stress at the introducer attachment feature to the nominal stress in the shell.

The influence of the shell manufacturing and processing was incorporated in the endurance limit value $\left(S_{e}\right)$. A baseline endurance limit for the appropriate Ti-6Al-4V alloy of $500 \mathrm{MPa}$ was used [31] for the machined cases (conditions a and d), and multiplied by a factor of 0.58 to account for the strength reduction caused by grit blasting and plasma-spray coating with HA [17], giving an endurance limit of $S_{e}=290 \mathrm{MPa}$ for conditions b and c. In all cases the minimum yield strength required by this material's standard [32] of $S_{y t}=760 \mathrm{MPa}$ was used.

\subsection{Fatigue Analysis Methodology B: Low Cycle Fatigue Loads}

The Soderberg approach was used as a conservative criterion to allow design without fatigue damage accumulation under normal, high cycle fatigue loads. However, this approach cannot account for the effects of higher magnitude, low cycle fatigue loads or occasional overloads upon fatigue life. These effects could considerably influence fatigue life through load sequence effects, if damage is accumulated or residual stress is generated by yield. Therefore, low cycle fatigue load events were considered simply, and conservatively, by comparing the material's yield strength to the peak stress under overload conditions, with appropriate stress concentrations applied. 
In the ceramic bearing insert, assembly was predicted to generate an axisymmetric residual tension field with a peak

First Principal stress of 31.0 MPa on its external surface, over which the service loads superimposed a local patch of biaxial tension (Figure 4 top). In gait this generated a peak tensile stress of up to $178 \mathrm{MPa}$ at $40^{\circ}$ inclination, and in stumbling this generated up to $233 \mathrm{MPa}$ (Figure 5 top). The interference fit generated approximately $112 \mathrm{MPa}$ of compression in the shell rim, which protected this thinner section of the shell against excessive tension at steeper inclinations. Therefore in high cycle fatigue the worst case was predicted to occur at $40^{\circ}$ inclination, where the position of the residual and service loading stress concentrations coincided, and were approximated to a cycle of $\sigma_{\text {mean }}=135 \mathrm{MPa}$ and $\sigma_{\text {amp }}=66.2 \mathrm{MPa}$ (or stress ratio $R_{S}=0.26$ for a load ratio $R_{L}=0.1$ ). The peak low-cycle event stress was $233 \mathrm{MPa}$, or $28 \%$ of the material's tensile strength.

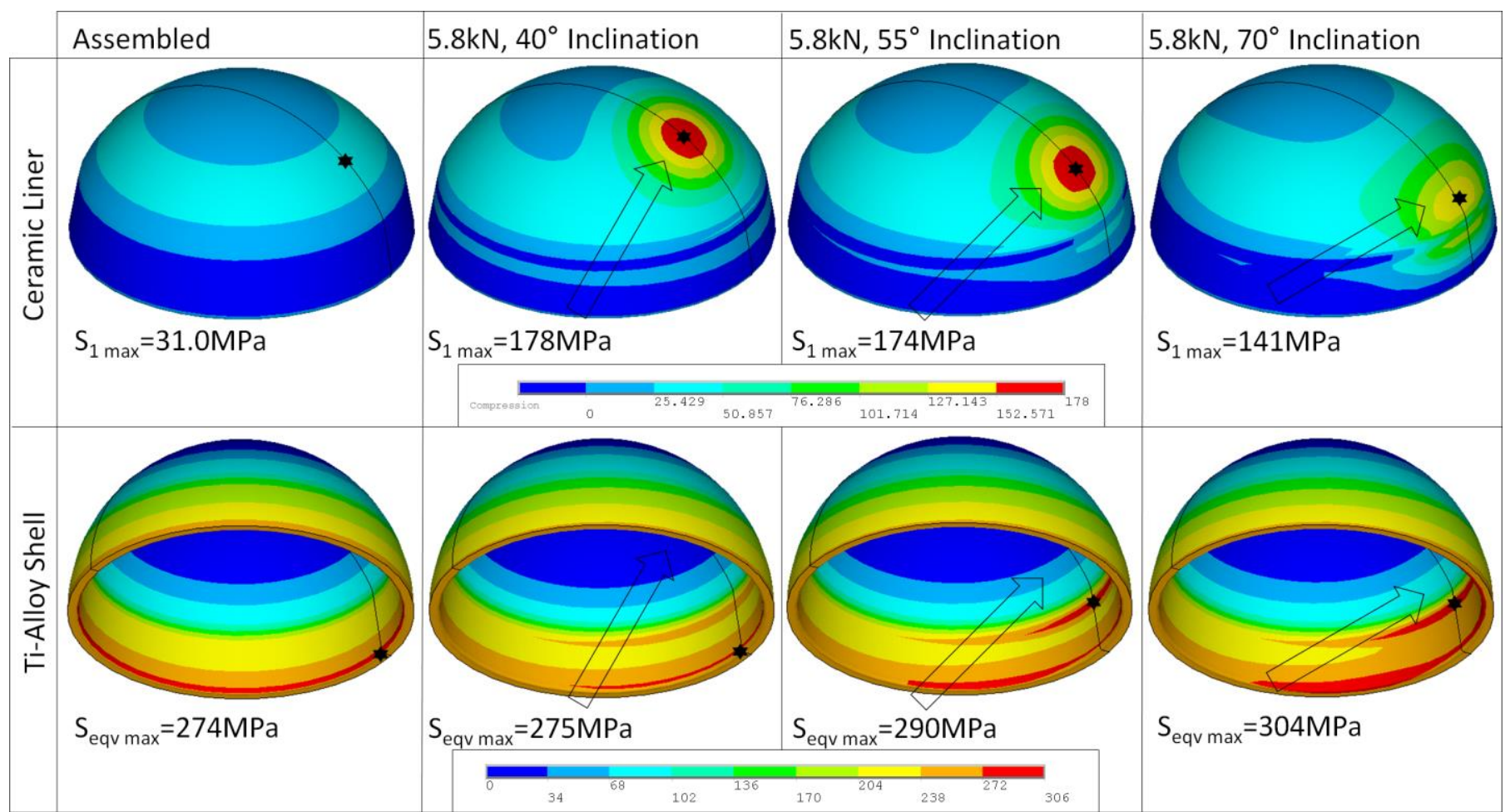

Figure 4: $1^{\text {st }}$ Principal Stress in the Ceramic Bearing Insert (top) and Equivalent Stress in the Titanium Alloy Shell (bottom) under 5.8kN Gait Loading Arrow indicates Load Vector, Star indicates Peak Location.

In the titanium alloy shell, assembly generated axisymmetric residual hoop tension with a peak of $274 \mathrm{MPa}$ at the shell rim (Figure 4 bottom). The contact pressure on the taper interface under assembly and loading conditions was inspected, and positive contact was predicted around the full periphery of the band under all loading conditions. This indicated that under standard service loads, the interference fit would be sufficient to avoid disassembly of the cupinsert structure. The peak stress under service loading was located on the internal, machined surface, predicted as $304 \mathrm{MPa}$ at $70^{\circ}$ inclination, and at this location to the predicted cyclic stresses were calculated as $\sigma_{\text {mean }}=274 \mathrm{MPa}$ and $\sigma_{\text {amp }}=30.5 \mathrm{MPa}$ (or stress ratio $R_{S}=0.80$ for a load ratio $R_{L}=0.1$ ).

Considering high cycle fatigue, these raw equivalent stress field data for the titanium alloy shell were used to calculate the cyclic mean stress and stress amplitude for each in-vivo load case, incorporating geometric stress concentrations and worst-case error margins of model verification. These were used to calculate the reserve factor vs. the Soderberg infinite life limit for each node in the model, using an appropriate endurance limit for the surface in question (Figure 6). The worst case of high cycle fatigue in all measures occurred when the cup was loaded as if inclined at $70^{\circ}$. The highest cycle mean stress was $335 \mathrm{MPa}$ on the internal surface, and $314 \mathrm{MPa}$ on the external surface, both at the shell rim. The highest stress amplitude was $44.3 \mathrm{MPa}$ on the internal surface at the taper interface, and 53.6 MPa at the corresponding height on the external surface (Figure 6). The minimum reserve factors were calculated as $N_{f}=1.96$ on the internal surface and 1.96 on the external surface, on the taper interface and on the shell's coated external surface near the rim, respectively. Considering low cycle fatigue, the peak cyclic stress in the titanium shell under the $11 \mathrm{kN}$ traumatic load case was $366 \mathrm{MPa}$ (Figure 5 bottom), or $48 \%$ of the material's yield strength, a safety factor of 2.08 . 


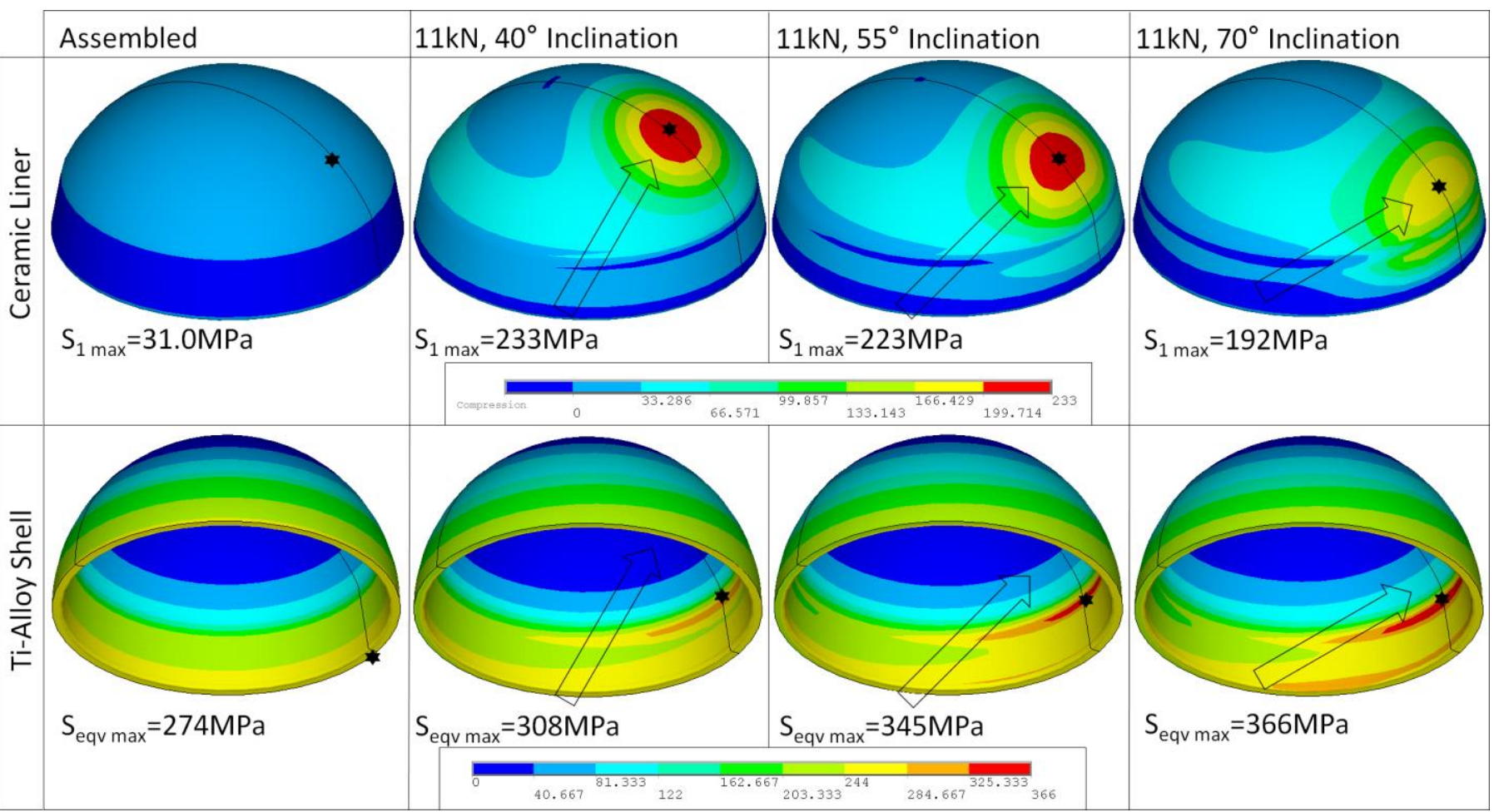

Figure 5: $1^{\text {st }}$ Principal Stress in the Ceramic Bearing Insert (top) and Equivalent Stress in the Titanium Alloy Shell (bottom) under 11kN Stumbling Loading Arrow indicates Load Vector, Star indicates Peak Location.

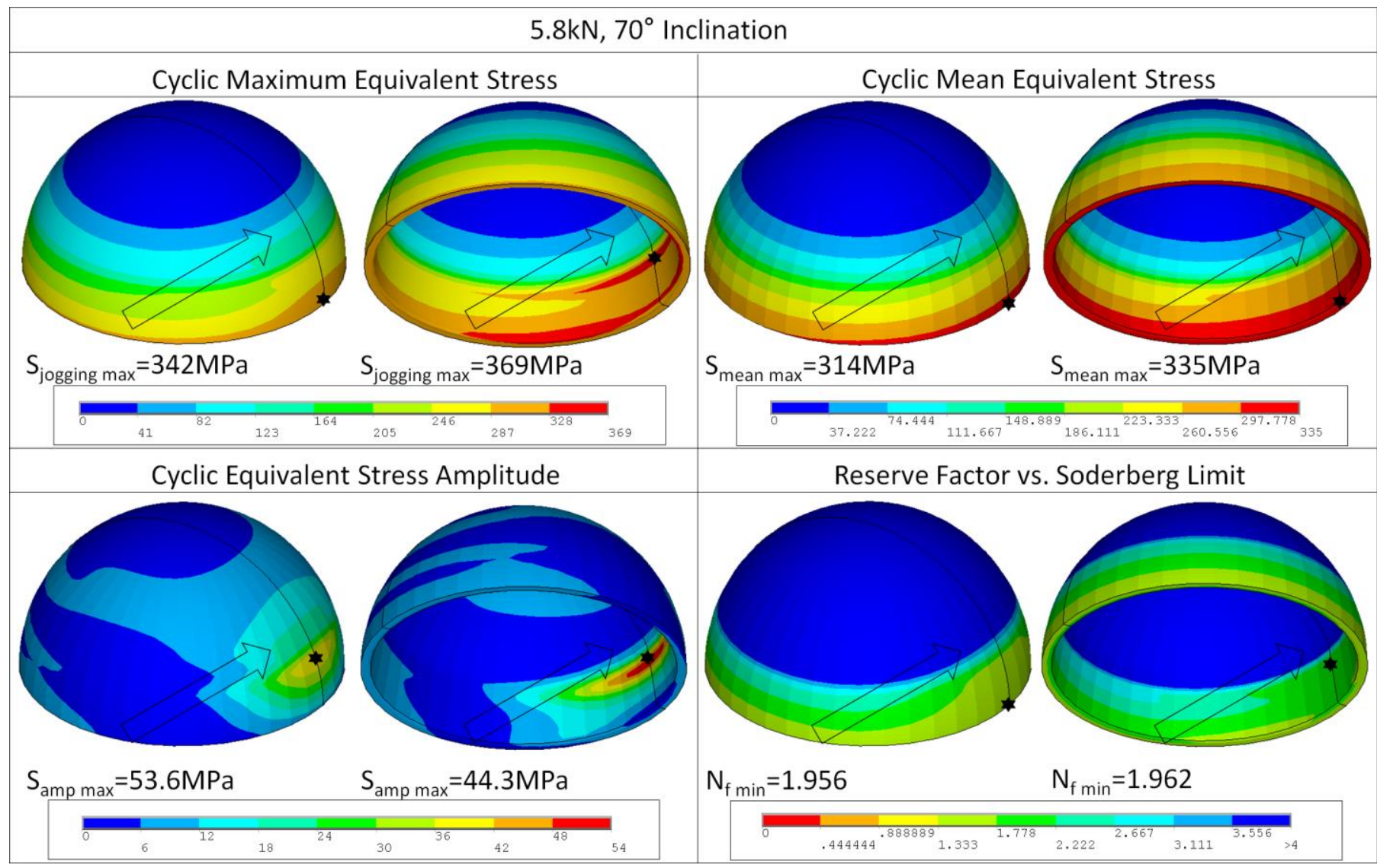

Figure 6: Fatigue Cycle Maximum Stress (top left), Mean Stress (top right), Stress Amplitude (bottom left) and Reserve Factor vs. Soderberg Infinite Life Limit (bottom right) with $7^{\circ}$ Cup Inclination. Results shown on External and Internal Surfaces. Arrow indicates Load Vector, Star indicates Peak Location.

These results were corroborated by physical testing (Appendix 2). 


\section{DISCUSSION}

In this study, a fatigue strength assessment methodology for the pre-clinical analysis of modular assembled implants was demonstrated, using a case-study prototype thin-walled modular acetabular cup, analysed under assembly and in-vivo loading.

Predictions from computational analysis suggested that in both implant components, the peak tensile stresses would be insuffic ient to cause static failure under traumatic loading. The peak ceramic insert tensile stress was $233 \mathrm{MPa}$, in biaxial flexion, 3.56 times below the material's estimated $830 \mathrm{MPa}$ tensile strength. This value was based on a reported flexural strength of $1384 \mathrm{MPa}$ [33] and the typical 60\% ratio of the tensile strength to the flexural strength for this material type [18]. The peak titanium alloy shell equivalent stress was $366 \mathrm{MPa}, 2.08$ times below its 760 $\mathrm{MPa}$ lower bound yield strength. This was used as verification of the cup under low cycle fatigue conditions.

Fatigue analysis under worst-case high cycle loads, representing jogging, considered the mean and cyclic stresses in comparison to the conservative Soderberg infinite life limit. This took account of the influence of cup manufacturing methods upon material endurance, and the implicit influence of stress concentrating design features such as fins and introducer attachment points. For the case study cup concept, this approach predicted reserve factors above 1.96 for the titanium alloy shell, over a range of loading directions.

Considering the ceramic bearing component, published fatigue strength data for the BIOLOX delta ceramic material (CeramTec GmbH, Plochingen, Germany) was sparse. One reported study assessed its fatigue life under extreme mechanical and environmental conditions for biomechanical applications, and observed no fatigue failures and no reduction in static strength after 20 million $300 \mathrm{MPa}$ flexion fatigue cycles and hydrothermal aging equivalent to 20 years in-vivo [34]. Their conditions were more extreme than the predicted stresses in this cup concept.

Fatigue assessment using a single in-vivo FE analysis and the Goodman, Gerber and Soderberg limits has precedence in pre-clinical analysis of existing and conceptual mono-block implants [35, 36]. The modified approach used in this study, employing full-field analysis of stresses both from residual and superimposed in-vivo loads was justified where the fatigue stress ratio $R_{s}$ may be considerably larger than the fatigue load ratio $R_{l}$ in pre-stressed modular implants. As a result it was observed that in pre-stressed modular cups, with high residual stress in the titanium alloy shell, the locations where the peak cyclic mean stress and peak cyclic stress amplitude were experienced did not correspond with each other, or with the minimum reserve factor location (Figure 6). Conversely, the ceramic component demonstrated a more conventional low stress ratio, as its residual tensile stresses are lower, so its peak cyclic stresses corresponded with its overall peak stress (Figure 4).

A key step in the verification of design concepts using this methodology would be the collection of case-specific yield strength and endurance limit data at the appropriate $R_{s}$ ratio. This would be collected for each of the modelled surface conditions, employing the relevant material processing methods, treatments and coatings. A mechanical test arrangement for collecting case-specific data to add confidence to the technique could include series testing of machined and coated material finishes, and should be conducted in a representative environment such as Ringers solution (Figure 2e). It should be noted that this study aims to present the justification of a general fatigue strength evaluation process for modular implants, rather than to present a testing and analysis process for acetabular cups in particular. Researchers aiming to employ this process should conduct their own, case-specific material strength characterisation and model validation exercises.

The simulations and tests are subject to several limitations. The true clinical scenario that these models aim to describe is highly non-deterministic, subject to variability in loading, surgical positioning and the mechanical properties of the supporting bone and its interface with the implant. Moreover, the geometry of the cup components is not constant, with each dimension varying across a manufacturing tolerance range. This will have a considerable effect upon the elastic energy stored in the taper interface and the transmission of stress into the supporting material. Confidence in the computational analysis could be increased with the consideration of these variables through probabilistic methods. However, there is no theoretical limit to the range and magnitude of traumatic loads which can be imposed upon orthopaedic implants, so worst-case test methods and conservative pass criteria must be defined. In this example, worst realistic case normal, non-microseparating loading (high cycle fatigue) and occasional overloading (low cycle fatigue) scenarios were simulated, and the results were analysed using conservative endurance limit, yield strength, stress concentration factor and notch sensitivity factor values. This approach is conservative and will lead to some additional over-engineering, but it negates the effects of load 
sequence interactions for the loading regime considered, and is safer for the orthopaedic implant application where no fatigue damage accumulation may be tolerated. Even worse cases of surgical positioning could occur, but it must be assumed that these rare cases would be detected during clinical follow-up, and corrected.

It is important to note that the analyses presented here represent part of a larger structural verification process: further testing and analysis would consider the effects of traumatic loading with micro-separation between the head and the cup, and dynamic bearing edge-loading. These adverse loading conditions may have little clinical consequence in thicker walled traditional designs [37] but could be of greater concern for thinner-walled implants. Furthermore, while the presented approach is justified for analys is of the risk of component structural failure through a single stress concentration, it may not be applicable for predicting progressive failure modes, or failures in the supporting bone and at fixation interfaces. For example, to predict fatigue micro-cracking of bone cement, or accumulated damage at a cementless implant-bone fixation interface, a different analys is approach is required, such as a cumulative fatigue or damage mode1 [38]. Fatigue strength evaluation is only one step in a broader development process, which should involve a series of verifications with the full range of normal and traumatic physiological loading scenarios, in a representative environment.

The models were also a necessary simplification of the clinical scenario, in order to avoid biasing the development of the novel prosthesis to a single pelvis. This involved the use of simplified support geometry and stiffness, and the re is a case to develop a generalised test setup for this purpose [39, 40]. Furthermore each model considered a single quasi-static load vector angle representing the peak joint contact force of the gait cycle. In an axisymmetric acetabular cup, in which the joint contact force primarily generates tensile stress, this can be accounted for by the analys is of a range of cup loading angles. However, the analysis of several quasi-static load cases may be necessary to analyse other implants such as femoral heads and stems, in which the range of joint contact force vector angles may generate anterior-posterior reverse bending. Bending and articulation generates cyclic tension and compression, which has long been recognised clinically and was a cause of failure of early femoral stems [41].

Further limitations of the modelling process relate to assumptions regarding the fixation between the implant and bone or support material. Researchers employing this technique will need to make assumptions regarding pressfitting of cementless fixed implants, the frictional characteristics, and degree of bonded fixation at the implant-bone interface. This was resolved in the current case-study by considering, in the fatigue stress analysis, additional stresses that would result from a worst-case of fixation (Section 2.4). This model represented a scenario in which the bearing frictional torque was sustained by a single fin, indicating complete de-bonding between the cup's fixation surface and the bone.

Care must be taken when using FE models to obtain absolute predictions of stresses, in particular where discontinuities of geometry or material properties produce stress concentrations. This is relevant to modular assemblies, where the edge of the contact interface can produce a geometric stress concentration. This factor is of particular importance with the presented methodology, which is a post-processing of absolute stress predictions. Stress results must be therefore be verified, and where possible the models validated, in both modular components. Stress also converges with mesh refinement more slowly than deflections and strain energy [42]. The presented technique requires accurate stress predictions and therefore thorough assessment of mesh convergence, especially at potentially stress concentrating edges of contact pairs. This may be achieved through local mesh refinement, use of p-method FE analys is, or in cases of divergent stress concentrations, the application of stress-concentration-limiting finite element methods [43]. This approach requires empirical, case-specific data which describes stress attenuation around stress raising geometry. A simplification of the model related to stress concentrations was the sub-modelling of the stress concentrating features in the titanium alloy shell. There is the potential that stress concentrating features in the shell could produce additional stress concentrations in the liner. This was predicted to be only a small effect with the present implant design, where all stress concentrating features were located in the outside of the metal shell, but should be considered when this process is applied to other designs.

The methodology presented here may have other applications, including the analysis of existing designs which demonstrate elevated clinical failure rates. The influence of cyclic stresses and wear upon damage at taper interfaces in modular femoral total hip implants has recently become a subject of particular concern. Clinical evidence suggests modular taper interfaces may be a source of metal ions, and hence have an influence upon metal sensitivity reactions [44]. This is also a region of sufficiently high stress to have caused clinical fatigue failures in primary [45] and revision replacements [46]. Furthermore, whilst bulk mechanical failure of mono-block femoral stems is now a very rare occurrence, there have been clinical reports of fractures of femoral stems with modular necks, linked to 
fatigue in Ti-6Al-4V [47] with the possible influence of fretting and corrosion [48, 49] and consistent with regions of high stress [50]. The approach presented in this study may be applied to understand these clinical failure modes, extended to incorporate the effects of fretting and corrosion in the endurance limit and stress concentration factors employed.

\section{CONCLUSIONS}

This study demonstrates a pre-clinical analysis method for modular orthopaedic implant designs under cyclic loading, for which the analysis of peak stresses alone may be insufficient. Computational analyses based on conservative input data and corroborated by physical tests indicated that a case study acetabular cup implant's structural integrity was sufficient to sustain extreme normal cyclic in-vivo loads (i.e. without impingement or microseparation), when correctly implanted and when malpositioned. The methods illustrate the importance of considering modular assembly residual stresses upon an implant's strength, alongside stress concentrating effects arising from manufacturing processes and geometric features. Fatigue strength evaluation is only one step in a broader development process, which should involve a series of verifications with the full range of normal and traumatic phys iological loading scenarios, with representative boundary conditions and a representative environment. This study presents and justifies a fatigue analysis methodology which could be applied in early stage development of a wide range of novel prosthesis designs, and is particularly relevant as implant designs aim to conserve bone at the expense of reduced safety factors compared to over-engineered traditional designs.

\section{ACKNOWLEDGEMENTS}

The authors would like the acknowledge CeramTec GmbH, Sandvik Medical Solutions Ltd., Jossi AG and Medicoat AG for providing test specimens, and Miss Katherine Wilson for her assistance with mechanical testing.

\section{DECLARATIONS}

Competing Interests:

None Declared.

\section{Funding:}

This research was funded by the University of Southampton, Finsbury Development Ltd., and Aurora Medical Ltd.

\section{Ethical Approval: \\ Not Required.}


According to Soderberg [29], the reserve factor against high cycle fatigue damage accumulation under a defined loading scenario giving non-zero mean stress was calculated using Equation 1:

$$
N_{f}=\left(\frac{K_{f \times} \sigma_{a m p}}{s_{\varepsilon}}+\frac{\sigma_{\text {mean }}}{s_{y t}}\right)^{-1}
$$

where:

$K_{f}=$ the fatigue stress concentration factor,

$\sigma_{\text {amp }} \quad=$ the cyclic stress amplitude,

$\sigma_{\text {mean }}=$ the mean stress,

$S_{e} \quad=$ the material's endurance limit,

$S_{y t} \quad=$ the material's yield strength, and

$N_{f} \quad=$ the resulting predicted reserve factor.

Each node's cyclic mean stress and stress amplitude were calculated from the residual $\left(\sigma_{\text {assembly }}\right)$ and peak cyclic $\left(\sigma_{\text {jogging }}\right)$ load cases, accounting for the fatigue load ratio $R_{L}$. The peak cyclic stress $\left(\sigma_{\text {jogging }}\right)$ contains contributions both from the applied joint contact load and the assembly pre-load. The mean stress (Eq.2a) was calculated using the cycle stress at maximum 'stance' load $\left(\sigma_{\max }\right.$, Eq.2b) and at minimum 'swing' load ( $\sigma_{\min }$, Eq.2c). As the maximum stress could occur under the maximum or the minimum load, due to the assembly's pre-stress, the stress amplitude was calculated as the modulus of the half-difference between stance and swing load stresses (Eq.2d):

$$
\begin{aligned}
& \sigma_{\text {mean }}=\frac{\left(\sigma_{\max }+\sigma_{\min }\right)}{2} \\
& \sigma_{\text {max }}=\sigma_{\text {jogging }} \\
& \sigma_{\min }=\sigma_{\text {assembly }}+R_{L} \times\left(\sigma_{\text {jogging }}-\sigma_{\text {assembly }}\right) \\
& \sigma_{\text {amp }}=\left|\frac{\left(\sigma_{\max }-\sigma_{\min }\right)}{2}\right|
\end{aligned}
$$

The influence of stress concentrating geometric features in high cycle fatigue was incorporated in the fatigue stress concentration factor $\mathrm{K}_{\mathrm{f}}$, calculated by Equation 3:

$$
K_{f}=1+q\left(K_{t}-1\right)
$$

where $q$ is the notch sensitivity factor $(0-1)$ and $K_{t}$ is the stress concentration factor $(\geq 1)$. A notch sensitivity factor for Ti-6Al-4V of $q=0.44$ was used [51], appropriate for the present scenario's predicted fatigue stress ratio $\left(R_{s}\right.$, obtained from the FE results). 
All FE model meshes were verified with convergence analyses considering peak stress values (implant model) and peak deflections (support models). Preliminary FE analyses produced comparable stress distribution predictions for the hemi-pelvis and PMMA supported cups, and showed that the stiffer PMMA support produced a conservative case, with approximately $25 \%$ higher implant stresses. The more computationally efficient PMMA supported model was used for the fatigue analysis, as it permitted a considerably finer mesh to be used, of particular importance for assessment of stress concentrations in the implant. Stresses in the titanium alloy structures remained below the material's yield strength, verifying the use of a linear elastic material model.

The coefficient of friction used in both models for the ceramic liner - titanium alloy shell interface was determined by empirical fit between the FE model and physical test assemblies. The axial press-in displacement of the liner relative to the shell was compared at a set assembly load, for coefficients of friction of $0.20,0.25,0.30,0.35$ and 0.40. For these C.O.F. values and nominal (mid-tolerance) dimension cup geometry, the predicted displacement was $115 \%, 107 \%, 101 \%, 96 \%$ and $91 \%$, respectively, of the mean of ten repeat physical test results. Therefore, by fitting a second order polynomial to the data, a C.O.F. of 0.32 was selected.

The PMMA-supported model was validated experimentally in terms of the generated residual cup stress, using uniaxial strain gauges to measure the circumferential strain at two locations in eight titanium shells. The circumferential stress was calculated from the strain data, and their mean value agreed with the FE analysis predictions to within $10 \%$. Therefore an error margin of $10 \%$ was incorporated in the fatigue analysis by applying a scaling factor of 1.21 to all stresses, compound ing the error margin for the two analyses. Validation of the PMMA supported model versus the hemi-pelvis support was not conducted.

For further verification combining the assembly and in-vivo loading, physical testing was conducted with prototype cups. Five cups were fatigue tested using an Instron 8878 axial servo-hydraulic machine (Instron Corp., Norwood, MA, USA. Capacity $25 \mathrm{kN}$ ). The cups were potted in aluminium cylinders with Technovit PMMA at an angle of $60^{\circ}$ to horizontal, and loaded with a compatible modular ceramic ball head between $0.580 \mathrm{kN}$ and $5.80 \mathrm{kN}$ (approximately $584 \%$ bodyweight, load ratio $R_{L}=0.1$ [21]) at a frequency $f=30 \mathrm{~Hz}$ for 10 million cycles. $60^{\circ}$ inclination was the steepest of the modelled loading angles which could be tested practically (Figure 2d), and is likely to be the most extreme clinical condition. Cups were oriented rotationally so that the stress concentrating introducer attachment features would align with the highest shell stress location, giving a worst case of positioning. A limitation was that frictional torque was not included in the fatigue test scenario described, so these effects would require verification with additional testing, for example on a hip simulator with appropriate support. Following fatigue testing, the cups were disassembled and the ceramic components were inspected using dye penetrant (Rocol Flawfinder ${ }^{\circledR}$, Rocol Ltd., Leeds, UK) to identify any surface damage. Finally, static burst tests were carried out on three additional, new cups inclined at $60^{\circ}$, using an Instron 1196 electromechanical test machine applying $0.5 \mathrm{kN} / \mathrm{s}$ ramped loading, until failure or a load of $100 \mathrm{kN}$ was achieved. No cups failed during 10 million fatigue cycles. No surface damage was observed on inspection using dye penetrant. No cups failed at $100 \mathrm{kN}$ static loading. One cup was loaded up to $187.5 \mathrm{kN}$, and failed due to fracture of the ceramic bearing insert. 


\section{REFERENCES:}

1. Bergmann, G., Deuretzbacher, G, Heller, M, Graichen, F, Rohlmann, A, Strauss, J, Duda, G N, Hip contact forces and gait patterns from routine activities. J Biomech, 2001. 34: p. 859-871.

2. Berg mann, G., Graichen, F, Rohlmann, A, Hip Joint Contact Forces during Stumbling. Langenbecks Arch Surg, 2004. 389: p. 53-59.

3. Schmalzreid, T.P., Szuszczewicz, E S, Northfield, M R, Akizuki, K H, Frankel, R E, Belcher, G, Amstutz, H C, , Quantitative Assessment of Walking Activity after Total Hip or Knee Replacement. J Bone Joint Surg, 1998. 80(1): p. 54-59.

4. Morlock, M.M., Schneider, E, Bluhm, A, Vollmer, M, Berg mann, G, Muller, V, Honl, M, Duration and Frequency of Every Day Activities in Total Hip Patients. J Biomech, 2001. 34: p. 873-881.

5. Silva, M., Shepherd, E F, Jackson, W O, Dorey, F J, Schmalzreid, T P, Average Patient Walking Activity Approaches 2 Million Cycles Per Year. J Arthoplasty, 2002. 17: p. 693-697.

6. McMinn, D., Treacy, R, Lyn, K, Pynsent, P, Metal-on-metal surface replacement of the hip. Clin Ortho Rel Res, 1996. 329S: p. S89-S98.

7. Dickinson, A.S., Browne, M, Jeffers, J R T, Taylor, A C, Analysis of a Thin Walled, Large Bore Ceramic Acetabular Cup with Improved in-vivo Stability and Integrity. Trans Orthopaedic Research Society, 2009. 55: p. 2399.

8. $\quad$ Tuke, M.A., Design Rationale for a Large Diameter Ceramic on Ceramic Hip Bearing. J Bone Joint Surg [Br], 2010. 92-B(Suppl. 1): p. 169.

9. Beaulé, P.E., Schmalzreid, T P, Udomkiat, P, Amstutz, H C, Jumbo Femoral Head for the Treatment of Recurrent Dislocation Following Total Hip Replacement. J Bone Joint Surg [Am], 2002. 84-A: p. 256-263.

10. Peters, C.L., McPherson, E, Jackson, J D, Erickson, J A, Reduction in Early Dislocation Rate with Large-Diameter Femoral Heads in Primary Total Hip Arthroplasty. J Arthoplasty, 2007. 22(6 Suppl. 2): p. 140-4.

11. Venditolli, P.A., Lavigne, M, Girard, R, Roy, A G, A randomised study comparing resection of acetabular bone at resurfacing and total hip replacement. J Bone Joint Surg [Br], 2006. 88-B: p. 997-1002.

12. Miller, A.N., Su, E P, Bostrom, M P G, Nestor, B J, Padgett, D E, Incidence of Ceramic Liner Malseating in Trident Acetabular Shell. Clin Orthop Rel Res, 2009. 467: p. 1552-1556.

13. Langdown, A.J., Pickard, R J, Hobbs, C M, Clarke, H J, Dalton, D J N, Grover, M L, Incomplete Seating of the Linear with the Trident Acetabular System. J Bone Joint Surg [Br], 2007. 89-B: p. 291-295.

14. Cook, S.D., Thomas, K A, Fatigue Failure of Noncemented Porous-Coated Implants. J Bone Joint Surg [Br], 1991. 19: p. 20-24.

15. Long, M., Rack, H J, Titanium Alloys in Total Joint Replacement - a Materials Science Perspective. Biomaterials, 1998. 19: p. 1621-1639.

16. Dick, J.C., Bourgeault, C A, Notch Sensitivity of Titanium Alloy, Commercially Pure Titanium, and Stainless Steel Spinal Implants. Spine, 2001. 26: p. 1668-1672.

17. Evans, S.L., Gregson, P J, The Effect of a Plasma-Sprayed Hydroxyapatite Coating on the Fatigue Properties of Ti-6Al4V. Materials Letters, 1993. 16: p. 270-274.

18. MatWeb.MatWeb Website, www.matweb.com. Material Property Data. August 2013].

19. Sawbones. Sawbones Website, www.sawbones.com. January 2010].

20. Kulzer, H., Technovit 3040 Data Sheet:Heraeus GmbH, Hanau, Germany.

21. Berg mann, G., Graichen, F, Rohlmann, A, Hip joint loading during walking and running, measured in two patients. J Biomech, 1993. 26: p. 969-990.

22. Bergmann, G., Graichen, F, Rohlmann, A, Bender, A, Hein lein, B, Duda, G N, Heller, M O, Morlock, M M, Realistic Loads for Testing Hip Implants. Bio-Medical Materials and Engineering, 2010. 20(2): p. 65-75.

23. Robinovitch, S.N., Hayes, W C, McMahon, T A, Prediction of Femoral Impact Forces in Falls on the Hip. J Biomech Eng, 1991. 113: p. 366-374.

24. Majumder, S., Roychowdhury, A, Pal, S, Simulation of Hip Fracture in Sideways Fall using a 3D Finite Element Model of Pelvis-Femur-Soft Tissue Complex with Simplified Representation of Whole Body. Med Eng and Physics, 2007. 29: p. 1167-1178.

25. Majumder, S., Roychowdhury, A, Pal, S, Effects of Trochanteric Soft Tissue Thickness and Hip Impact Velocity on Hip Fracture in Sideways Fall through 3D Finite Element Simulations. J Biomech, 2008. 41: p. 2834-2842.

26. Teno, J., Kiel, D P, Mor, V, Multiple Stumbles: a Risk Factor for Falls in Community-Dwelling Elderly. A Prospective Study. J AM Geriatr Soc, 1990. 38(12): p. 1321-1325.

27. Gerber, H., Bestimmung der zulassigen Spannungen in Eisenkonstructionen. Zeitschrift des Bayerischen Architekten und Ingenieur-Vereins, 1874. 6: p. 101-110.

28. Goodman, J., Mechanics Applied to Engineering. 1899, London: Longman, Green \& Company.

29. Soderberg, C.R., Factor of Safety and Working Stress. Trans ASME, 1930. 52.

30. Scholes, S.C., Unsworth, A. Tribological Testing of Pre-assembled Alumina Matrix Composites Hip Prostheses. in Bioceramics and Alternative Bearings in Joint Arthroplasty, 13th Biolox Symposium. 2010. Edinburgh, UK: Springer.

31. Chao, J., Lopez, V, Failure Analysis of a Ti6Al4V Cementless HIP Prosthesis. Engineering Failure Analysis, 2007. 14: p. 822-830.

32. ASTM, F163-12a: Standard Specification for Wrought Titanium-6Aluminium-4Vanadium ELI Alloy for Surgical Implant Applications. 2012. 
33. CeramTec, GmbH, BIOLOX delta - Nanocomposite for Arthroplasty: Scientific Information and Performance Data. 2011: p. MT0800 11-GB-2.000-0802.

34. Kuntz, M. Live-time prediction of BIOLOX delta. in Ceramics in Orthopaedics: 12th BIOLOX Symposium. Bioceramics and alternative bearings in joint arthroplasty. 2007. Seoul, Korea: Steinkopf Verlag.

35. Zafer Senalp, A., Kayabasi, O, Kurtaran, H, Static, Dynamic and Fatigue Behaviour of Newly Designed Stem Shapes for Hip Prosthesis using Finite Element Analysis. Materials and Design, 2007. 28: p. 1577-1583.

36. Pekedis, M., Yildiz, H, Comparison of Fatigue Behaviour of Eight Different Hip Stems: a Numerical and Experimental Study. J Biomedical Science and Engineering, 2011. 4: p. 463-650.

37. Esposito, C.I., Walter, W L, Roques, A, Tuke, M A, Zicat, B A, Walsh, W R, Walter, W K, Wear in Alumina-onAlumina Ceramic Total Hip Replacements. J Bone Joint Surg [Br], 2012. 94-B: p. 901-907.

38. Verdonschot, N., Hu iskes, R, The Effects of Cement-Stem Debonding in THA on the Long-Term Failure Probability of Cement. J Biomech, 1997. 30: p. 795-802.

39. Jin, Z.M., Meakins, Morlock, Parsons, Hardaker, Flett, Isaac, Deformation of press-fitted metallic resurfacing cups. Part 1: experimental simulation. Proc IMechE H, 2006. 220: p. 299-309.

40. Yew, A., Jin, Z M, Donn, A, Morlock, M M, Is aac, G, Deformation of press-fitted metallic resurfacing cups. Part 2: finite element simulation. Proc IMechE H, 2006. 220: p. 311-319.

41. Gruen, T.A., McNeice, G M, Amstutz, H C, "Modes of Failure" of Cemented Stem-Type Femoral Components. Clin Orth Rel Res, 1979. 141: p. 17-27.

42. Marks, L.W., Gardner, T N, The Use of Strain Energy as a Convergence Criterion in the Finite Element Modelling of Bone and the Effect of Model Geometry on Stress Convergence. J Biomed Eng, 1993. 15: p. 474-476.

43. Stolk, J., Verdonschot, N, Huiskes, R, Management of Strain Fields around Singular Points: Comparison of FE Simulations and Experiments, in, in Proc. 4th International Symposium on Computer Methods in Biomechanics and Biomedical Engineering, J. Middleton, Jones, M N, Pande, G N, Editor. 1999, Gordon and Breach. p. 57-62.

44. Langton, D.J., Jameson, S S, Joyce, T J, Gandhi, J N, Sidaginamale, R, Mereddy, P, Lord, J, Nargol, A V F, Accelerating Failure Rate of the ASR Total Hip Replacement. J Bone Joint Surg [Br], 2011. 93-B: p. 1011-1016.

45. Trigkilidas, D., Anand, A, Ibe, R, Syed, T, Floyd, A, A Fracture through the Neck of a Charnley Elite-Plus Femoral Component: a Case Report. Int J Orthop Surg, 2010. 16.

46. Bos, P.K., van Biezen, F C, Weinans, H, Femoral Component Neck Fracture after Failed Hip Resurfacing Arthroplasty. J Arthoplasty, 2011. 26: p. 1570 e1-e4.

47. Wilson, D.A.J., Dunbar, M J, Amirault, J D, Farhat, Z, Early Failure of a Modular Femoral Neck Total Hip Arthroplasty Component. J Bone Joint Surg [Am], 2010. 92: p. 1514-1517.

48. Dangles, C.J., Altstetter, C J, Failure of the Modular Neck in a Total Hip Arthroplasty. J Arthoplasty, 2010. 25: p. 1169 e5-e7.

49. Grupp, T.M., Weik, T, Bloemer, W, Knaebel, H-P, Modular Titanium Alloy Neck Adapter Failures in Hip Replacement - Failure Mode Analysis and Influence of Implant Material. BMC Musculoskeletal Disorders, 2010. 11.

50. Gill, I.P.S., Webb, J, Sloan, K, Beaver, R J, Corrosion at the Neck-Stem Junction as a Cause of Metal Ion Release and Pseudotumour Formation. J Bone Joint Surg [Br], 2012. 94-B: p. 895-900.

51. Naik, R.A., Lanning, D B, Nicholas, T, Kallmeyer, A R, A Critical Plane Gradient Approach for the Prediction of Notched HCF Life. Int J Fatigue, 2005. 27: p. 481-492. 